\title{
Miniaturized Flow Sensor with Planar Integrated Sensor Structures ON SEMICIRCUlar SURFACE CHANNELS
}

\author{
M. Dijkstra, M.J. de Boer, J.W. Berenschot, T.S.J. Lammerink, R.J. Wiegerink, M. Elwenspoek \\ MESA $^{+}$Research Institute, University of Twente, The Netherlands
}

\begin{abstract}
A calorimetric miniaturized flow sensor was realized with a linear sensor response measured for DI water flow from $40 \mathrm{nl} \cdot \mathrm{min}^{-1}$ up to $300 \mathrm{nl} \cdot \mathrm{min}^{-1}$. A versatile technological concept is used to realize a sensor with thermally-isolated freely-suspended silicon-nitride microchannels directly below the substrate surface. The microchannel concept allows for the planar integration of sensor structures in close proximity to the fluid. Chemical-resistant fluidic connections can be made directly on top of the microchannels, without introducing large dead-volumes. The realized flow sensor consists of a microchannel with low hydraulic resistance and small total fluid volume.
\end{abstract}

\section{INTRODUCTION}

Fluidic applications within the field of micro- and nanofluidics often require the accurate measurement of small fluid flows, in the order of $\mathrm{nl} \cdot \mathrm{min}^{-1}$. For such small flow rates, standard bulk micromachined flow channels are not suitable, because a large part of the flow is in thermal contact with the substrate and does not contribute to the sensor signal. Instead, thermally isolated channels are required. To this end, a number of authors [1-3] have proposed freely suspended surface micromachined channels where the channel is realized by removing a sacrificial layer. A disadvantage of these sensors is the large hydraulic resistance of the microchannel, which is largely determined by the channel height being limited to only a few $\mu \mathrm{m}$. Additionally, the channel height can cause step-coverage problems for sensor structures required to be deposited across the microchannel.

This paper presents a miniaturized flow sensor applying freely suspended silicon-nitride semicircular microchannels. The hydraulic resistance can be freely chosen by adjusting the microchannel diameter. The microchannels are readily made below the substrate surface, allowing for the planar integration of sensor structures.

\section{DESIGN}

Figure 1 illustrates the design of the calorimetric miniaturized flow sensor. The sensor measures liquid flowing through a freestanding microchannel crossing a thermal-isolation cavity over a length of $800 \mu \mathrm{m}$. Fluidic interfacing to the microchannel is made possible by directly applying O-rings or capillary tubing connectors on top of the microchannel inlet and outlet imbedded in the substrate surface. The $5 \mathrm{~mm}$ long microchannel has $4.5 \mathrm{nl}$ total volume and a hydraulic resistance as low as $6.17 \mathrm{kPa} / \mu \mathrm{l} \cdot \mathrm{min}^{-1}$ for water. Fluid flow is measured by heating the fluid using a heater centered on the suspended microchannel. Heat conduction through the surrounding air, the microchannel and the electrical connection leads results in a symmetric temperature profile around the heater. The temperature profile is disturbed by forced convection on applying fluid flow through the microchannel. The disturbance does not affect the temperature of the heater for small flows, because there is no temperature gradient at the heater for the undisturbed symmetric temperature profile, required for the convection of heat.

The temperature disturbance is measured differentially by measuring the resistance change of two resistors $40 \mu \mathrm{m}$ up- and downstream of the heater. For small flows the sensor output is linearly proportional to the flow rate, resulting in a highly sensitive calorimetric flow sensor. The differential sensor output is insensitive to commonmode ambient temperature fluctuations and variations in fluid temperature [4].

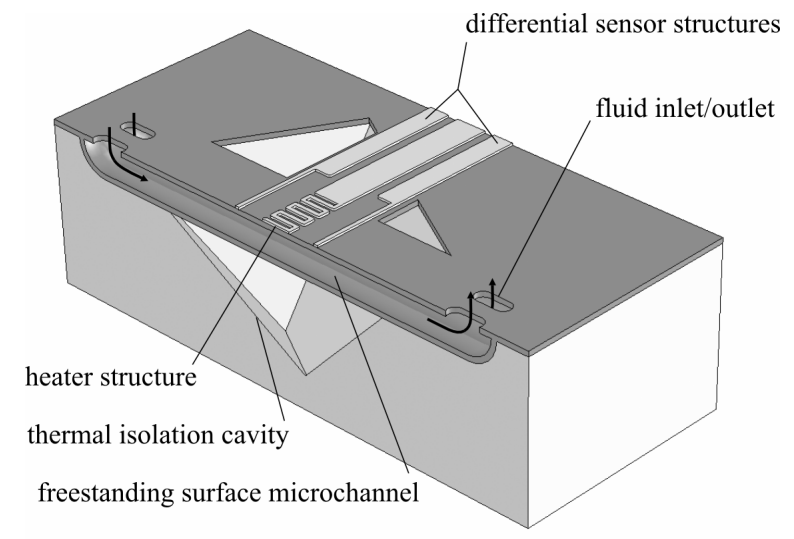

Figure 1: Cross-section of calorimetric flow sensor design.

The disturbed temperature profile upon increased fluid flow causes a gradient in temperature across the heater, leading to heat-loss at the heater, eventually decreasing the heater temperature, with the sensor output becoming non-linear.

\section{FABRICATION}

The fabrication of the thermal flow sensor is based on buried channel technology [5]. Buried channel technology is a good alternative, compared to surface micromachined channels, in realizing mechanically strong freely suspended microchannels, without the requirement for a sacrificial layer. The technology can be further simplified by making channels directly below the substrate surface $[6,7]$. This allows for the integration of sensor structures in close proximity to the fluid, with improved fluidic interfacing to the microchannels. 
The surface microchannels are created by isotropic dry etching, using high-density $\mathrm{SF}_{6}$ plasma with zero selfbias, through small etch holes in a low-stress $500 \mathrm{~nm}$ $\mathrm{Si}_{\mathrm{X}} \mathrm{N}_{\mathrm{Y}}$ layer [6]. The coalescence of the etched surfaces results in semicircular channel profiles (figure 2a), with the possibility to merge multiple parallel channel profiles.
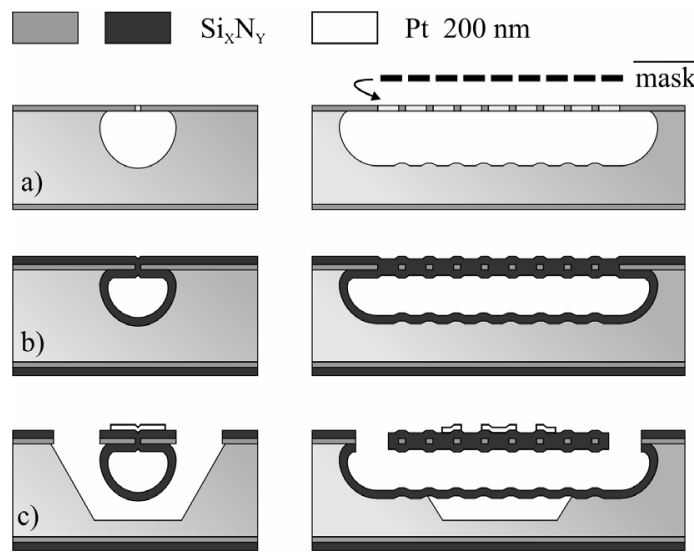

Figure 2: Process scheme for the fabrication of surface channel thermal flow sensor, with Pt sensor structures.

The small etch holes and the inner surfaces of the microchannels are conformally coated by the LPCVD deposition of a second low-stress $1.2 \mu \mathrm{m} \quad \mathrm{Si}_{\mathrm{X}} \mathrm{N}_{\mathrm{Y}}$ layer (figure 2b). Thus creating completely sealed microchannels, while leaving a planar substrate surface for further processing (figure 4). The semicircular channels are mechanical strong and are insensitive to deformation, possibly caused by the integrating of sensor materials with high stress across the microchannel.

Because of the resulting planar substrate surface, step coverage problems are avoided and $200 \mathrm{~nm}$ thin-film Pt sensor structures can be deposited directly on top of the microchannels (figure 2c). The $\mathrm{Pt}$ is patterned by oxidation using $\mathrm{O}_{2}$ plasma, with the thin oxidation layer acting as a mask during Aqua Regia etching [8], creating well defined sensor structures (figure 3).

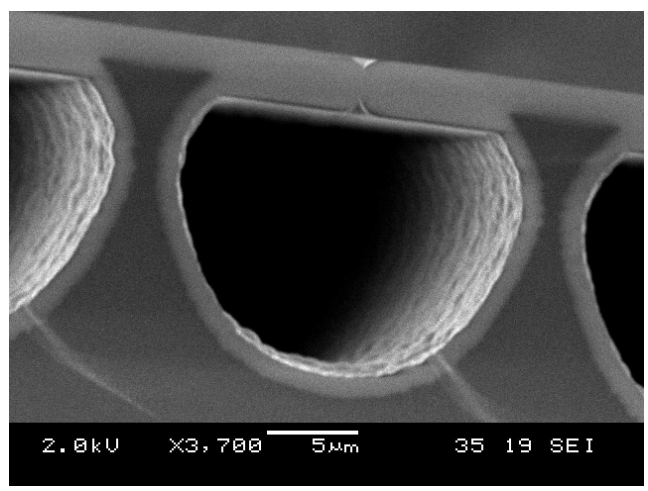

Figure 4: Cross-section of sealed surface channel, leaving planar substrate surface for further processing [6].

Fluid inlets/outlets are etched through the $\mathrm{Si}_{\mathrm{X}} \mathrm{N}_{\mathrm{Y}}$ layer (figure 2c) allowing for the direct interfacing to the microchannels on the frontside of the wafer. Thermalisolation cavities are defined using the same mask (figure 2c) and are etched by $\mathrm{KOH}$ etchant, suspending the surface microchannels above the heat conducting substrate (figure 3).

\section{SENSOR MODELING}

The temperature field surrounding the heater was solved with CFDRC using a high fidelity mesh of the sensor. The thermal field was solved numerically for the freely suspended microchannel containing fluid, the electrical connection leads and the surrounding air in the isolation cavity as well as part of the air above the sensor. A heating power of $1.9 \mathrm{~mW}$ was imposed on the heater and boundary conditions were set for the substrate to reside at ambient temperature. A laminar flow profile was solved inside the microchannel for various water flow rates, visualizing the disturbance on the temperature field by forced convection due to the fluid flowing through the surface microchannel.

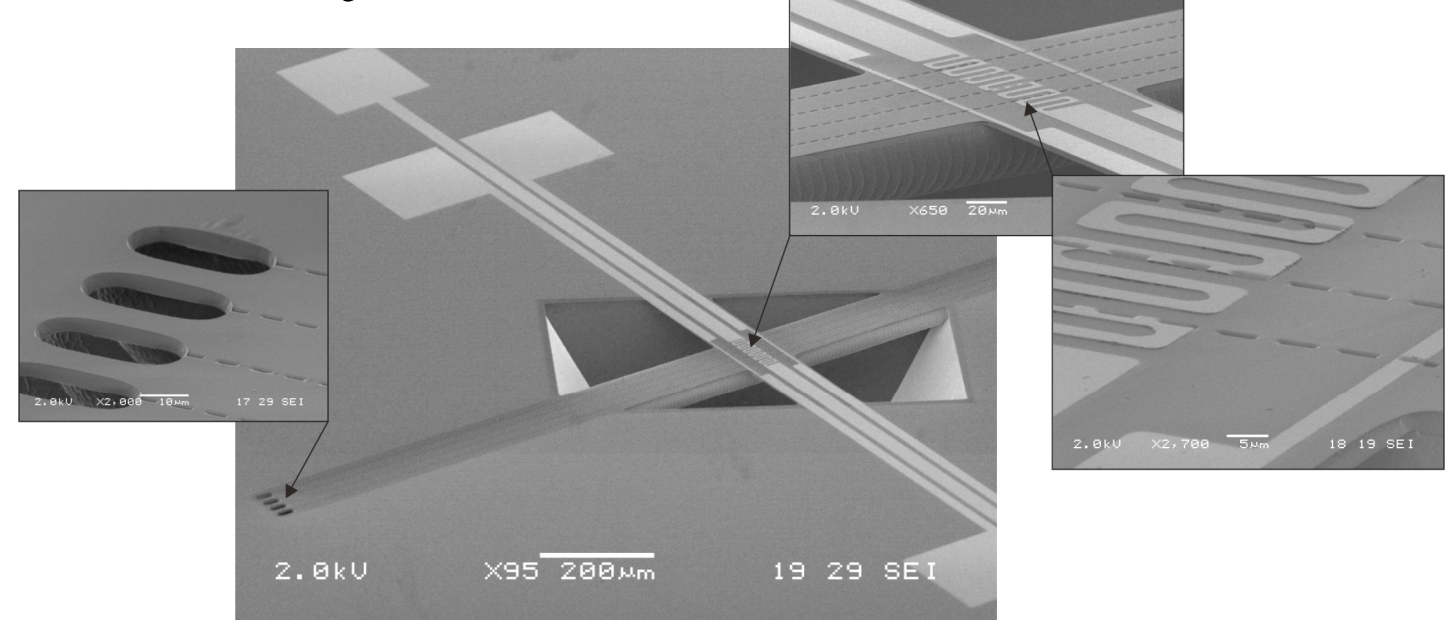

Figure 3: From left to right; Fluid inlet/outlet to the microchannel, allowing for the direct interfacing to the microchannel on the substrate surface. Calorimetric flow sensor suspended above thermal-isolation cavity. Pt resistors crossing microchannel deposited on planar substrate surface. Proper step coverage of $200 \mathrm{~nm}$ thin-film Pt across sealed microchannel etch holes. 


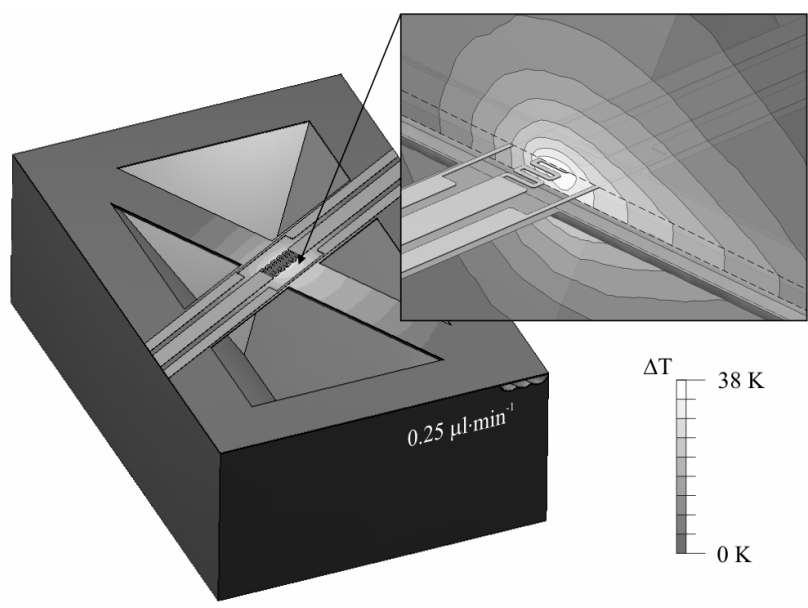

Figure 5: Numerical model of the sensor, showing asymmetric temperature profile along the surface channel at $0.25 \mu \mathrm{l} \cdot \mathrm{min}^{-1}$ DI water flow. The close-up shows the temperature field in a plane through the microchannel.

Figure 5 visualizes the calculated asymmetric temperature field on the freely suspended microchannel due to $0.25 \mu \mathrm{l} \cdot \mathrm{min}^{-1}$ water flowing through the microchannel. The close-up near the heater provides a view inside the microchannel. It clearly shows that the temperature field extends into the surrounding air due to heat conduction. Inside the microchannel the fluid is fully heated to a uniform temperature for a given distance from the heater. The forced heat convection is thus dependent on the total volume flow through the microchannel. The temperature gradient near the heater at a larger flow rate of $2 \mu \mathrm{l} \cdot \mathrm{min}^{-1}$ is observed to change rapidly to the lateral uniform temperature gradient inside the microchannel within $40 \mu \mathrm{m}$ from the heater.

The numerical model was solved for various water flow rates from $0 \mu \mathrm{l} \cdot \mathrm{min}^{-1}$ up to $2 \mu \mathrm{l} \cdot \mathrm{min}^{-1}$. Figure 6 shows the resulting temperature profiles on the freely suspended surface microchannel with the heater centered at $0 \mu \mathrm{m}$. As expected, the heater temperature for small flows is hardly affected and the disturbance by the flow increases the temperature downstream from the heater as

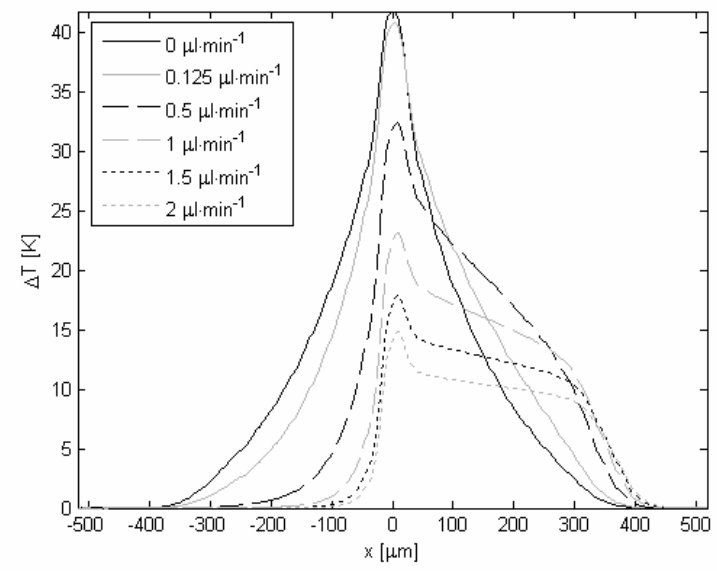

Figure 6: Temperature profiles along the microchannel, at various flow rates. The microchannel is fixed to the substrate at $\pm 400 \mu \mathrm{m}$. much as it decreases the temperature upstream. At higher flow rates the temperature profiles decrease in overall temperature, with a rapid decrease in temperature between $300 \mu \mathrm{m}$ and $400 \mu \mathrm{m}$, because there the microchannel is in close proximity to the thermal-isolation cavity.

\section{EXPERIMENTS}

Measurements were performed using a pressure driven flow setup as indicated in figure 7. DI water was forced through the microchannel by applying a pressure from a $\mathrm{N}_{2}$ gas cylinder. The flow rate was determined for each measurement point by measuring the advancing meniscus in the fluidic connection tube, which contains $493 \mathrm{nl} \cdot \mathrm{mm}^{-1}$, after applying a constant pressure for several minutes. The flow was kept constant by regulating a membrane valve to a fixed pressure in the range from 0 to 1 bar.



Figure 7: Schematic of experimental setup.

DI water flow was measured by applying a current $I_{\text {htr }}$ of $5 \mathrm{~mA}$ to the heater resistor dissipating $1.9 \mathrm{~mW}$. The resistance change of the heater upon fluid flow is measured and can be related to the temperature change of the heater according to $\Delta R=\alpha \cdot \Delta T \cdot R_{0}$, with $\alpha=$ $0.0024 \mathrm{~K}^{-1}$ the measured temperature coefficient of resistance and $R_{0}$ the resistance value at ambient temperature. The sensor resistors are placed in a Wheatstone bridge, applying a voltage $V_{\text {scr }}$ of $0.1 \mathrm{~V}$, where a differential output voltage $\Delta V$ is obtained, which is nearly linear proportional to the resistance change of both sensor resistors.

Figure 8a shows the measured resistance change compared to the change in temperature of the heater obtained from the numerical model for DI water flow rates from $0 \mu \mathrm{l} \cdot \mathrm{min}^{-1}$ up to $2 \mu \mathrm{l} \cdot \mathrm{min}^{-1}$. Figure $8 \mathrm{~b}$ shows the simultaneously measured differential voltage output of the Wheatstone bridge compared to numerical results. As expected, the heater temperature is nearly constant at small flow rates with a linear sensor response $\Delta V$ having a flow sensitivity of $S_{V}=218 \mu \mathrm{V} / \mu \mathrm{l} \cdot \mathrm{min}^{-1}$. The sensor output remains linear up to a flow rate of $300 \mathrm{nl} \cdot \mathrm{min}^{-1}$.

The experimental and numerical results are in close agreement. However, $R_{0}$ of the heater and sensor resistors cannot be determined accurately since a Kelvin resistance measurement is not possible. Therefore, the values of $R_{0}$ are chosen such that the experimental results are scaled to fit the numerical data. Obtained values of $R_{0}$ are close to expected values calculated from mask layout and sheet resistance data. 

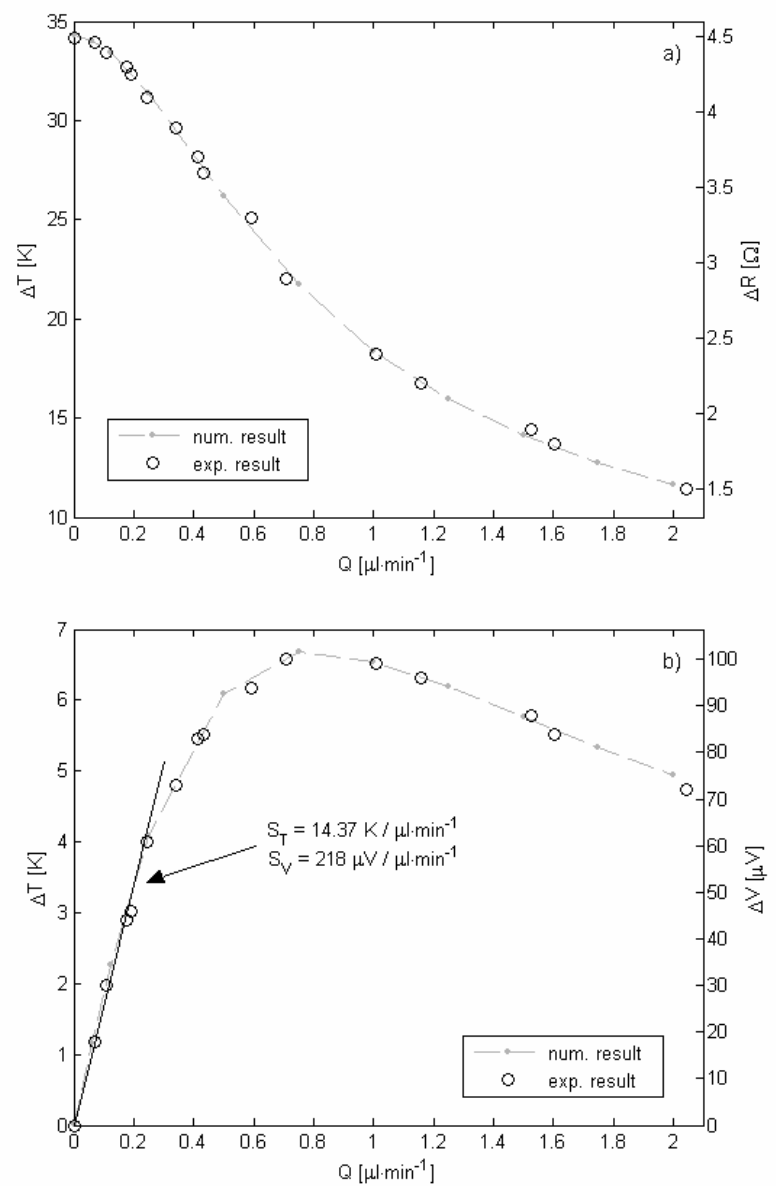

Figure 8: a) Temperature of the heater obtained from the numerical model compared to measured resistance change of the heater resistor. b) Differential temperature between both sensor resistors obtained from the numerical model compared to measured voltage output of the Wheatstone bridge.

Figure 9 shows additional measurements for DI water flow below $300 \mathrm{nl} \cdot \mathrm{min}^{-1}$. A fluid flow restriction was used in the measurement setup for flow rates below $100 \mathrm{nl} \cdot \mathrm{min}^{-1}$, allowing to measure at higher pressures.

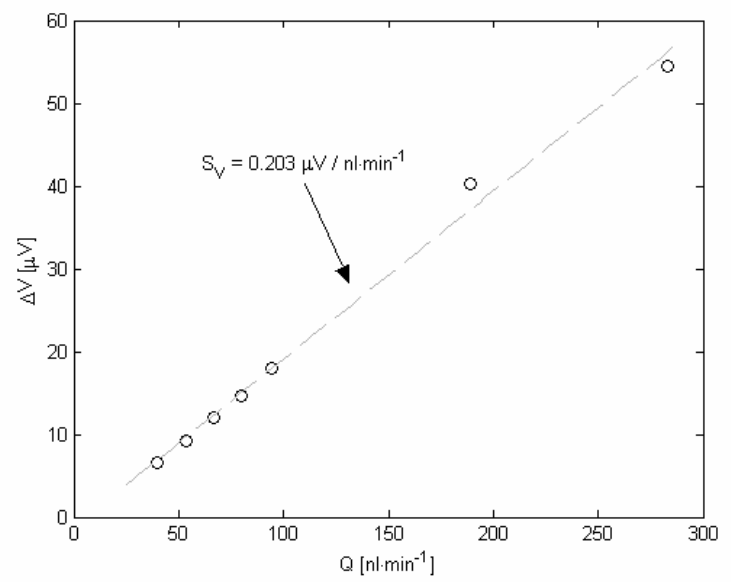

Figure 9: Linear sensor output for DI water flow below $300 \mathrm{nl} \cdot \mathrm{min}^{-1}$, with $\tau_{\text {sample }}=1 \mathrm{~s}$.
The flow rate becomes unstable for pressures approaching zero, which is currently the limiting factor in measuring liquid flow below $40 \mathrm{nl} \cdot \mathrm{min}^{-1}$.

\section{CONCLUSIONS}

A miniaturized calorimetric flow sensor was developed using a versatile surface channel concept. A linear differential calorimetric sensor response was measured for DI water flow from $40 \mathrm{nl} \cdot \mathrm{min}^{-1}$ up to $300 \mathrm{nl} \cdot \mathrm{min}^{-1}$. Up to a certain extent, the nonlinear response at higher flow rates can be corrected for by operating the heater at constant temperature. The surface microchannel technology allows for further experiments to be carried out on sensor materials, like materials with high $\alpha[1,2]$ or thermopiles.

\section{ACKNOWLEDGEMENT}

The authors would like to thank the Dutch Technology Foundation (STW) for financial support.

\section{REFERENCES}

[1] S. Wu, Q. Lin, Y. Yuen, Y-C. Tai, "MEMS Flow Sensor for Nano-Fluidic Applications”, Proceedings of MEMS, 2000

[2] Y. Mizuno, M. Liger, Y-C. Tai, "Nanofluidic Flowmeter using Carbon Sensing Element”, Proceedings of MEMS, 2004

[3] M. Dijkstra, T.S.J. Lammerink, R.J. Wiegerink, M. Elwenspoek, "Nano-Flow Thermal Sensors Applying Dynamic $\omega-2 \omega$ Sensing Method”, Proceeding of MME, 2006

[4] N.R. Tas, T.S.J. Lammerink, P.J. Leussink, J.W. Berenschot, H-E. de Bree, M. Elwenspoek, "Towards Thermal Flowsensing with pL/s Resolution", Micromachined Devices and Components VI, Proceedings of SPIE, Vol. 4176, 2000

[5] M.J. de Boer, R.W. Tjerkstra, J.W. Berenschot, H.V. Jansen, G.J. Burger, J.G.E. Gardeniers, M. Elwenspoek, A. van den Berg, "Micromachining of Buried Micro Channels in Silicon”, Journal of Microelectromechanical Systems, Vol. 9, No. 1, 2000

[6] M. Agah, K.D. Wise, “A Fully-Dry PECVDOxynitride Process for MicroGC Column Fabrication”, Proceeding of MEMS, 2005

[7] M. Dijkstra, M.J. de Boer, J.W. Berenschot, T.S.J. Lammerink, R.J. Wiegerink, M. Elwenspoek, "Versatile Surface Channel Concept for Microfluidic Applications”, Proceedings of MME, 2006

[8] M.J. Kim, L.A. Gruenke, R.J. Saia, S.S. Cohen, "Inhibition of Acid Etching of Pt by Pre-Exposure to Oxygen Plasma", Applied Physics Letters, Vol. 44, No. 4, 1984 